\title{
Numerical Investigation into the effects of obstacles on heavy gas dispersions in the atomsphere
}

\author{
Abdullah Alakalabi ${ }^{1, *}$, and Weiming Liu ${ }^{1}$ \\ ${ }^{1}$ School of Engineering, University of Central Lancashire, Preston, Lancashire, the UK, PR1 2HE
}

\begin{abstract}
Computational fluid dynamics (CFD) approach is applied to investigate heavy gas dispersion in the atmosphere, under the action of wind. Because of the effect of buoyancy, steady double peaks of the heavy gas concentrations in the downstream area are observed from the numerical results. The double peaks of the concentrations are a special pattern of heavy gas dispersion, which cannot be found in the neutral gas dispersions. Four types of obstacles are placed behind the leakage source to study the influences of these obstacles to the heavy gas dispersions. The numerical results show the detailed shapes and other contents of the heavy clouds under the obstacles.
\end{abstract}

\section{Nomenclature}

Abbreviations

CFD

ANSYS

2-D

3-D

$u$

$\rho$

mass $=\mathrm{M} / \mathrm{V}$

$\mathrm{p}$

$\tau$

$\mu_{t}$

$W$

$\Phi$

$R$

\section{Acronyms}

Computational Fluid Dynamics

Analysis System

Two Dimensional

Three Dimensional

Velocity vector

Density $\mathrm{kg} / \mathrm{m}^{3}$

Concentration $\mathrm{kg} / \mathrm{m}^{3}$

Pressure $\mathrm{Pa}=\mathrm{N} / \mathrm{m}^{2}$

shear Stress $\mathrm{Pa}=\mathrm{N} / \mathrm{m}^{2}=\mathrm{kg} / \mathrm{m} \mathrm{s}^{2}$

Turbulent Viscosity

Molecular mass of the gas mixture

Dissipation function

The universal gas constant

\section{Introduction}

Incident of chemical gas leakage is a serious safety and health hazard. To treat the incident properly and manage chemical gas storage correctly, we need to understand the behaviours of leaked gas dispersions. Experimental measurements and observations provide an intuitive knowledge on the dispersions. Noneless the experiments cannot produce the detailed processes. The numerical simulations can supplement the shortcomings of the experiments. Therefore, we apply the approach of computational fluid dynamics (CFD) to numerically investigate the heavy gas dispersions.

Our work is closely associated with the experimental results done by Ayrault et al. $(1994,1997)$ to produce a more complete understanding of the processes. The experiments investigated the negative buoyancy effects on the dispersion of continuous gas plumes with downwind obstacles. When the results of simulation are compared to experimental results, the buoyancy effects could be understood in greater detail.

For real-world safety applications, it is useful to understand the implications of accidental release of hazardous gases. Hazardous gases typically produce clouds, which are denser than the environment. In comparison to passive or positively buoyant plumes, negatively buoyant clouds behave differently. The negatively buoyant clouds are also sensitive to the effects of man-made and natural obstacles, which in turn, affect the dispersion. Thus, it is understandable that obstacles have a greater influence on heavy clouds when compared to passive buoyant plumes.

Density is a physical factor, which affects the inertia of the released materials. Close to the source, the influence is the most significant, because the density variation is very large there.

To significantly reduce the turbulence and turbulent mixing, it is critical to stably stratify the density variation. When the plume is stabilised, there is a reduction in plume depth, an increase in density effects, increase in width, and plume lateral dispersion. Since there is a density difference along the horizontal direction, there is an additional transport mechanism from the ambient flow. Thus, the

\footnotetext{
*Correspondence author: Abdullah Alakalabi, Email: aalakalabi@uclan.ac.uk
} 
lateral growth of a dense cloud results from the boundary layer turbulence and the buoyancy driven flow.

In the case of a dense plume spreading under a turbulent flow, some of the effects of plume stability include a reduction of plume depth, increase in density effects, increase of width, and plume lateral dispersion. This enables the plume to extend upwind of the source and for it to be wider than the size at the source. This phenomenon influences obstacle and shear stress effects. One of the industry applications involves quantifying and predicting these effects by defining different buoyancy flow parameters. The current understanding of dense cloud behaviour is based on many field and laboratory experiments.

Field experiments are based on realistic scenarios and scales for possible accidental releases of ammonia and hydrogen fluoride. One of the most significant challenges associated with field experiments is that many instruments are required, which make them expensive. Also, since the environmental conditions vary significantly from one day to another, it is challenging to quantify and analyse the results in a consistent manner. For this reason, most researchers and labs rely on laboratory experiments for simulating accidental releases. Wind tunnel and water channel experiments are common set-ups for most experiments. The main benefit of the approach is the ability to devise idealised laboratory experiments that allow for the investigation of specific phenomena, and specific physical processes could be investigated in greater detail and quantified.

It is also useful to recognise the limitations of these experiments. 2-D plumes or continuous plumes from an area source over flat terrain are away from realistic scenarios. The dispersion of accidentally released heavy gas plumes is influenced by obstacles near the source. An intuitive understanding of the presence of obstacles indicates that obstacles divert the flow, which increase mixing and dilute the plume in the absence of a channelling effect. Several studies have been performed to investigate the effects of obstacles on dispersion. These include 2-D fences and buildings that obstruct the dispersion of heavy plumes or jets. Other studies have investigated phenomena such as the transition of near-field heavy gas dispersion to far-field passive gas dispersion and the effect of the duration of the release on dispersion.

One of the valuable insights generated by the findings of these investigations is that the density difference and the densities of the ambient fluid and the cloud are not the only variables that affect the behaviour of the plume. More considerations including the interaction between the buoyancy parameters, characteristics of the external turbulent flow, initial release conditions including the size, shape, and discharge momentum are also necessary. When obstacles are present, specific geometries including 2- or 3D obstacles, heights, and the ratio between the height of the obstacle and the plume depth without the obstacle, and the angle become critical considerations. A significant amount of diligence is required for designing the studies and drawing meaningful interpretations from the results. This is primarily because experiments are idealised situations relating to the dispersion of continuous dense gas plumes. For design and modelling, it is useful to consider a variety of conditions to generate a better understanding of the effects of the associated phenomena.

Since obstacles in the real world could vary in shape volume, it is useful to consider the effects of some of the main characteristics of obstacles. Flat surfaces have been investigated extensively, and it is not in the scope of this investigation to discuss the results of flat surfaces. Obstacles could be considered as thin or thick obstacles such as fences and building blocks. Several studies have investigated turbulent flows around thin 2-D solid fences, which are typical in real-world scenarios.

The main finding associated with thin 2-D surfaces is that when a 3-D plume approaches a 2-D surface, the plume is blocked by the fence. The plume widens upstream and grows taller to surmount the fence with dilution. The buoyancy effect amplifies the increase of the lateral width, which is influenced by the plume jumping the obstacle.

The experiments indicate that in the case of dispersion problems, three parameters are critical for dispersion problems. The three parameters are the ratio of the fence height to the plume height at fence location, the Richardson number, and the ratio of the undisturbed plume height to the undistributed plume width at the obstacle location. The findings indicate that the shape of the fence is an important parameter, which is shown by the downstream vortices flow phenomenon, which is one of the effects of negative buoyancy on the external flow. This understanding is useful for modelling the influence of shape on dispersion. For the plume, the understanding helps characterise the geometrical evolution of plumes.

\section{Numerical methods}

The heavy gas dispersions involve in the gas flow and mixture with the ambient air. The flow is controlled by Navier - Stokes equations

$\frac{\partial \rho}{\partial t}+(u \cdot \nabla) \rho+\rho \nabla \cdot u=0$

$\rho \frac{\partial u}{\partial t}+\rho(u \cdot \nabla) u+\nabla p=\rho g+\mu \nabla \cdot\left\{\nabla u+(\nabla u)^{T}\right\}$

$\rho \frac{\partial e}{\partial t}+\rho(u \cdot \nabla) e+p(\nabla \cdot u)=\nabla \cdot(\lambda \nabla T)+\Phi$

where $u, \rho, T, p, e, \Phi$ and $g$ denote, respectively, the velocity vector, density, temperature, pressure, internal energy, dissipation function and gravity vector, while $\lambda$ and $\mu$ are the conduction coefficient and viscosity of the gas mixture, respectively. According to the thermodynamics we can get the relationship between the temperature and internal energy, $e=c_{v} T$, where $c_{v}$ is the specific heat capacity at constant volume.

$\overline{\text { *Correspondence author: Abdullah Alakalabi, Email: aalakalabi@uclan.ac.uk }}$ 
The mixing processes of the heavy gas and ambient air is controlled by transport equation

$\rho \frac{\partial Y_{i}}{\partial t}+\rho(u \cdot \nabla) Y_{i}=\nabla \cdot\left(\rho D_{i} \nabla Y_{i}\right)$

where $Y_{i}$ is the fraction of concentration for the species $\mathrm{i}$ and $D_{i}$ is its diffusion coefficient. Because of $\sum Y_{i}=1$ and the continuity equation $(1), \Sigma\left[\nabla \cdot\left(\rho D_{i} \nabla Y_{i}\right)\right]=0$ is required. The thermodynamically state equation is written by (5)

$p=\frac{\rho R T}{W}$

$W=\sum\left(\frac{Y_{i}}{W_{i}}\right)^{-1}$

where $R$ is the universal gas constant and $W$ is the molecular mass of the gas mixture which is calculated by (6). In equation (6), $W_{i}$ is the molecular mass of the species i. One can see the molecular mass of the mixture is not a constant. It will vary because of the mixing of the leaked gas with the surrounding air.

\subsection{Turbulence model}

The flows studied in this work are actually turbulent. The equations (1) - (6) are for the direct numerical simulations of turbulence that can produce detailed and very small scales structures of turbulence. In this work, however, we mainly concern the averaged behaviours of turbulence. Therefore, the equations to be solved are the Reynolds averaged Navier - Stokes equations. The Reynolds averaged Navier - Stokes equations involve in a set of unknown Reynolds stresses that reflect the effects of the unsolved and small-scale flows to the averaged quantities. In this work, we employ the $\kappa-\varepsilon$ turbulence model [1-2]. Thus, the Reynolds stresses have the same formulae as the viscous, but the turbulent viscosity is calculated by

$\mu_{t}=\rho c_{\mu} \frac{\kappa^{2}}{\varepsilon}$

where $\mu_{t}$ is the turbulent viscosity, $c_{\mu}=0.09$ is an empirical constant and $\kappa$ and $\varepsilon$ are calculated by two additional equations.

\subsection{Numerical schemes}

The software of ANSYS CFX is used to solve the equations above which adopts the finite volume method [3]. First of all, an unstructured mesh is generated over computational domain and node-central control volume is employed. The equations above are integrated over each control volume. Then the integrations are approximately calculated to result in a coupled system of algebraic equations. During the approximations, the second order high resolution scheme is applied for the flux calculations of the convective terms, to avoid the numerical instability.

The pressure-velocity coupling system is iteratively solved. Against the segregated method in which the pressure and velocity equations are separately calculated, the coupling method produces more accurate results.
The density of the mixture is not constant. It varies with the temperature change and produces a buoyancy. To capture this effect, an approximate relationship of the density and pressure is applied in each iteration,

$\rho_{i+1}=\rho_{0}+\frac{\partial \rho}{\partial p}\left(p_{i}-p_{0}\right)$

This is equivalent to the so-called low-Mach number simplification of the Navier - Stokes equations [4]. The benefit from that is significantly reducing the computational cost for unsteady flows.

\section{Setup of numerical modelling}

To investigate the influences of obstacles on heavy gas dispersions, a computationally geometric configuration is designed as Figure 1. Its dimension is $4 \mathrm{~m} \times 2 \mathrm{~m} \times 0.6 \mathrm{~m}$, respectively along the $\mathrm{x}, \mathrm{y}$, and $\mathrm{z}$ coordinate axes. This configuration is the same as the experimental research [5] so that the simulated results can be compared with it.

\section{Figure 1}

Computational Domain and Obstacle Arrangements

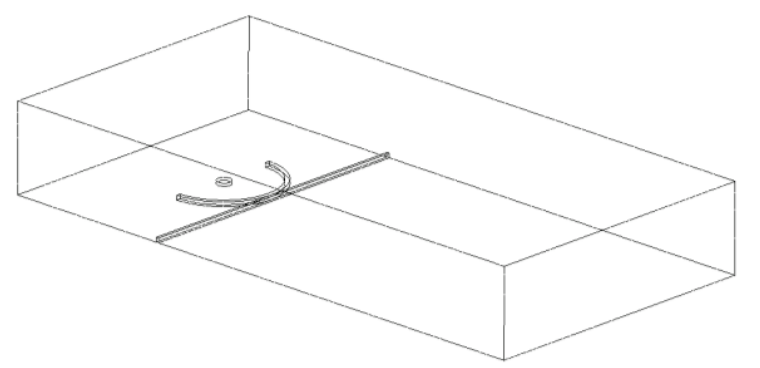

As we can see, there are two different solid obstacles. One is a straight fence at $400 \mathrm{~mm}$ downstream from the source point. The fence is $2 \mathrm{~m}$ long, i.e., equivalent to the width of the computational domain. This fence has two heights, $0.03 \mathrm{~m}$ or $0.06 \mathrm{~m}$. The other obstacle is a semi-circular fence with the radius of $0.4 \mathrm{~m}$ and the height of $0.03 \mathrm{~m}$. Combination of these obstacles forms five scenarios which is given in Table 1.

Table 1

Arrangement of the obstacles

\begin{tabular}{|c|c|c|c|}
\hline $\begin{array}{c}\text { Scenario } \\
\text { Number }\end{array}$ & \multicolumn{2}{|c|}{ Straight Fence } & $\begin{array}{c}\text { Semi-circular } \\
\text { Fence }\end{array}$ \\
\hline & $0.03 \mathrm{~m}$ & $0.06 \mathrm{~m}$ & $0.03 \mathrm{~m}$ \\
\hline 1 & No & No & No \\
\hline 2 & Yes & No & No \\
\hline 3 & Yes & No & Yes \\
\hline 4 & No & Yes & No \\
\hline 5 & No & Yes & Yes \\
\hline
\end{tabular}

An unstructured mesh is generated over the computational domain of Figure 1. This mesh is comprised of the hexahedral-dominant elements of about 3 millions. 
The ambient air has a standard atmospheric condition, i.e., its temperature is 25 degree of Celsius and the pressure is $101.3 \mathrm{kPa}$.

The leakage source is at the centre of the semi-circular fence. It is a small cylinder of $0.05 \mathrm{~m}$ diameter and $0.02 \mathrm{~m}$ height. The heavy gas leaks from its top face. The leaked gas velocity is $1.0 \mathrm{~m} / \mathrm{s}$. The density and temperature of the gas are $2.1 \mathrm{~kg} / \mathrm{m}^{3}$ and -100.0 degree of Celsius.

A wind inlet boundary condition is applied on the left-hand side of the domain, while the right-hand side has an outlet boundary. The symmetric boundary condition is applied to the other boundaries.

\section{Results and discussions}

The numerical simulation results are presented and discussed in these sections.

The molecular mass of the leaked gas is equal to that of the ambient air. Nonetheless, since the temperature of the leaked gas is significantly lower over the ambient air, the density of the leaked gas is much heavier than that of the ambient air. As a result, a heavy gas cloud is formed after the leakage.

In the initial stage, the dispersion of the leaked gas is unsteady. After the development of the unsteady dispersion, a steady heavy gas cloud is built up and stays there for long time.

For the steady states, Figure 2 shows the concentrations of the heavy gas of the 5 scenarios on the cross-section of $0.01 \mathrm{~m}$ away from the ground, of which Figure 2 (1) is the scenario without an obstacle. It is observed that the heavy gas disperses from left to right under the action of the wind and double peaks of the concentrations take shape. The double peaks of the concentrations are the special shape for the heavy gas dispersion. If the leaked gas has same density as the ambient air, the dispersion of the gas will make a single peak of its concentration staying in the middle area. These results are qualitatively consistent with the experimental observations [6].

The double peaks of the heavy gas concentrations are resulted from the buoyancy in the stratified flow. Because of the buoyancy, a bubble of the heavy gas is formed just behind the leakage source. This bubble is located at the centre of the domain, see Figure 2 (1). Existence of the bubble withstands the heavy gas transport in the central area, so that the heavy gas disperses along the two sides and the double peaks of the concentrations are build up eventually. All the processes are controlled by the buoyancy.

The other four graphics of Figure 2, i.e., Figure 2 (2) to (5), display the influences of the obstacles to the heavy gas dispersions. From Figure 2, one can see the five different shapes of heavy gas clouds. The reason for that is because of the presence of the different obstacles. First, comparing Figure 2 (1) with the others we see that the presence of obstacles will block the heavy gas dispersion to the downstream domain. Then, the different forms of obstacles will result in the distinct heavy gas shapes. The higher the fence, the more the heavy gas centralizes in the upstream. If a semi-circular fence exists, the upstream centralization of the heavy gas will be further enhanced.

Figure 2

Heavy gas concentrations of 5 scenarios on the horizontal crosssections of $0.01 \mathrm{~m}$ from the bottom boundary; the number under the figures correspond to the scenario number in Table 1.

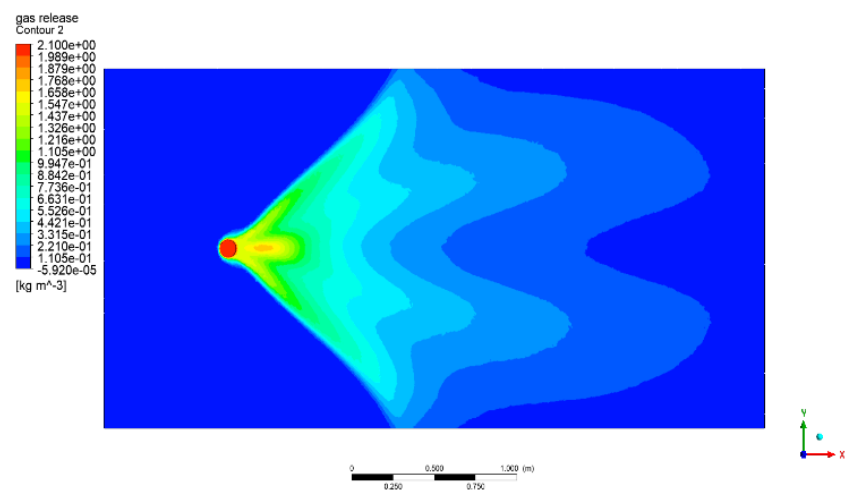

(1)

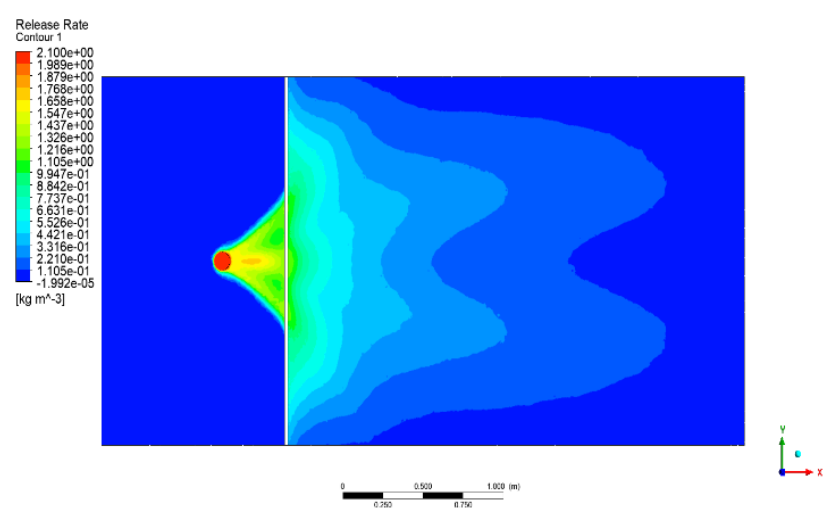

(2)

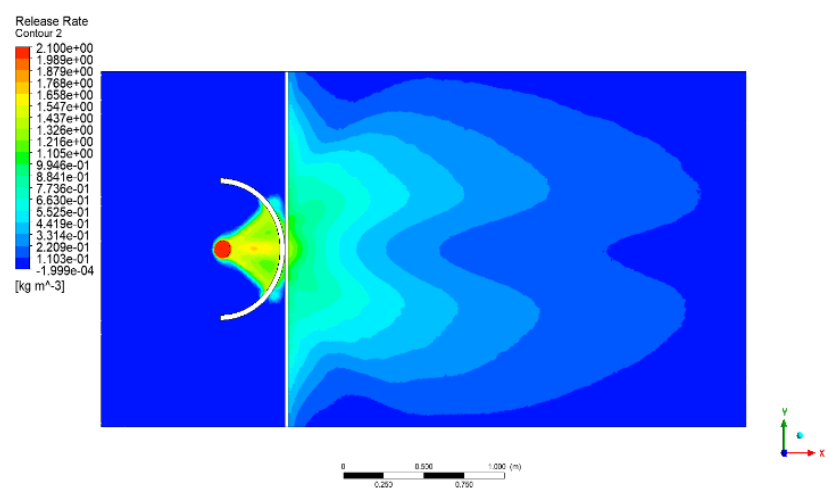

(3)

*Correspondence author: Abdullah Alakalabi, Email: aalakalabi@uclan.ac.uk 


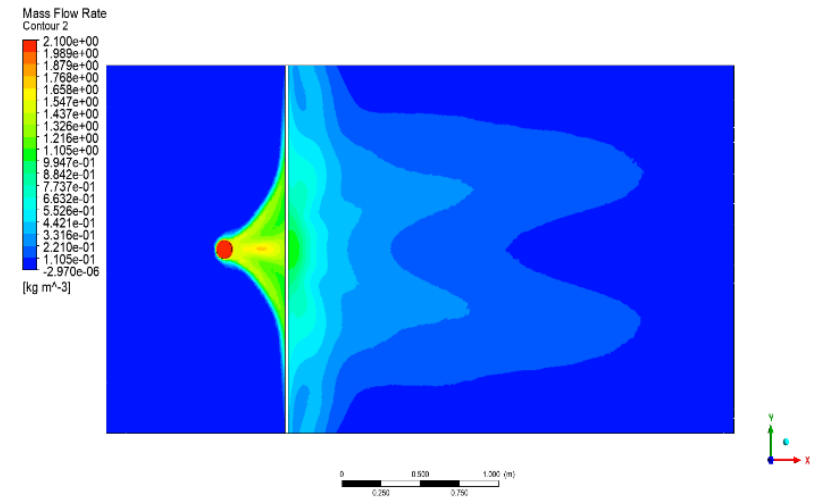

(4)

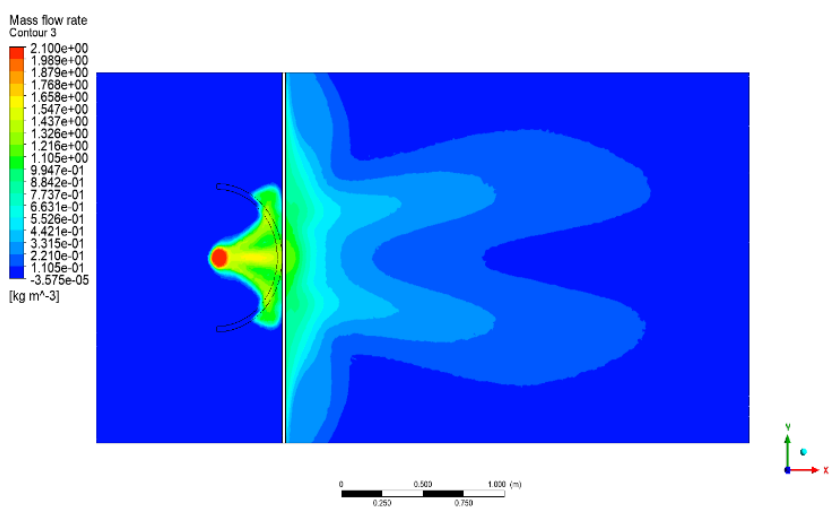

(5)

In order to quantitatively compare the numerical results with the experimental measurements [6], the crosswise and streamwise profiles of the heavy gas concentrations are illustrated in Figure 3. For the purpose of comparison, the measured data are transformed to the coordination's consistently with the numerical calculations. One can see that the numerical results are basically in agreement with the measured data.

Figure 3

Comparison with the experimental data, (a) crosswise profiles and (b) streamwise profiles

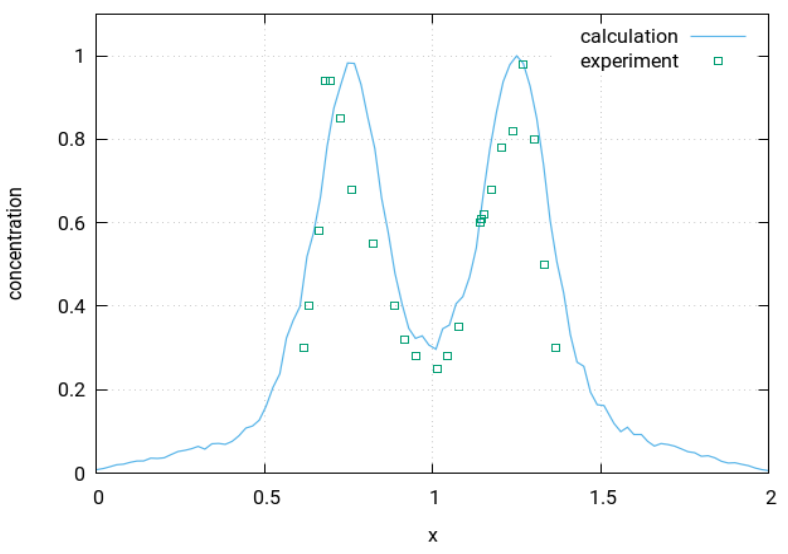

(a)

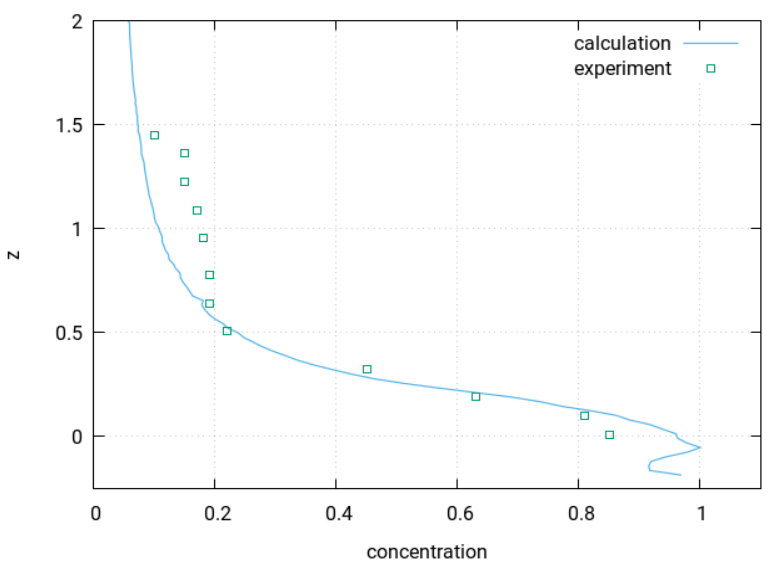

(b)

As discussed above, the buoyance plays an important role in heavy gas dispersions. In order to obtain the knowledge of the buoyance, Figure 4 illustrates the density of the mixture of the heavy gas and ambient air. The larger the density, the bigger the buoyance.

\section{Figure 4}

Densities of the mixture of the heavy gas and ambient air on the horizontal cross-sections of $0.01 \mathrm{~m}$ from the bottom boundary for 5 scenarios.

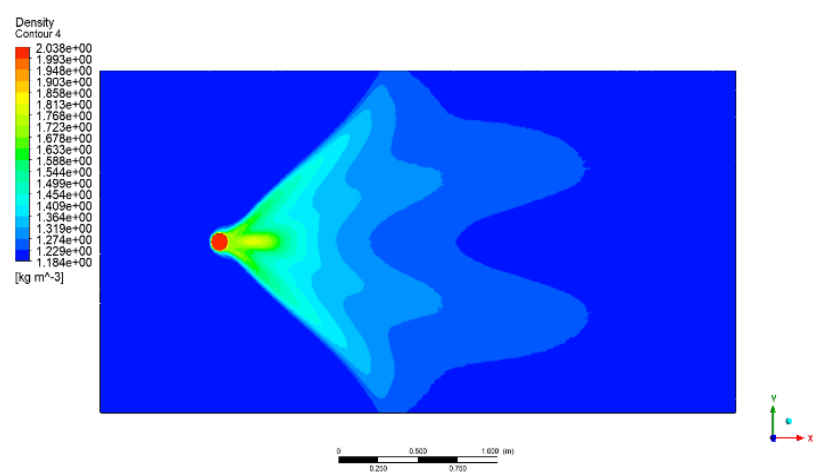

(1)

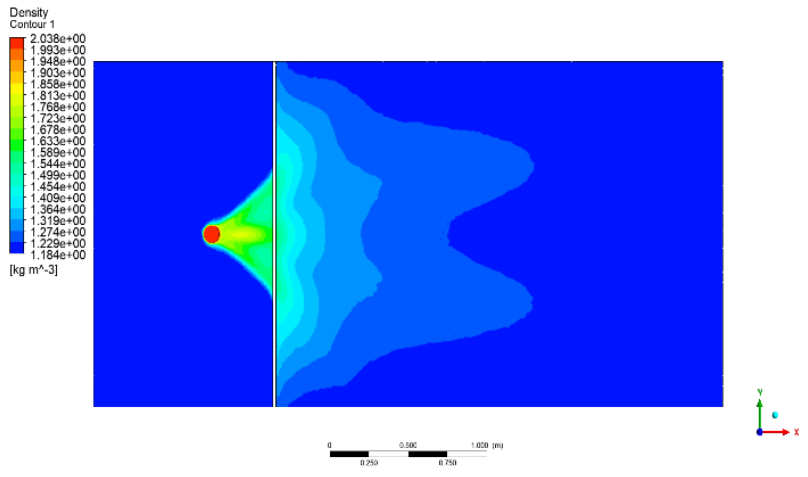

(2)

*Correspondence author: Abdullah Alakalabi, Email: aalakalabi@uclan.ac.uk 


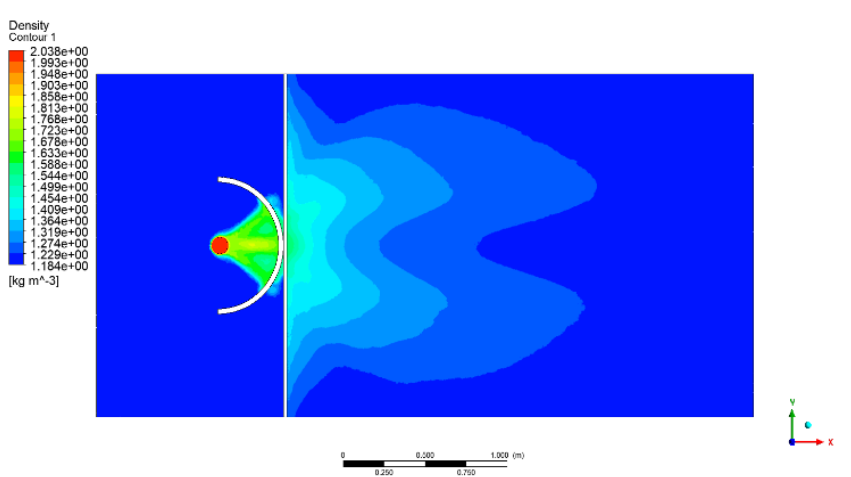

(3)

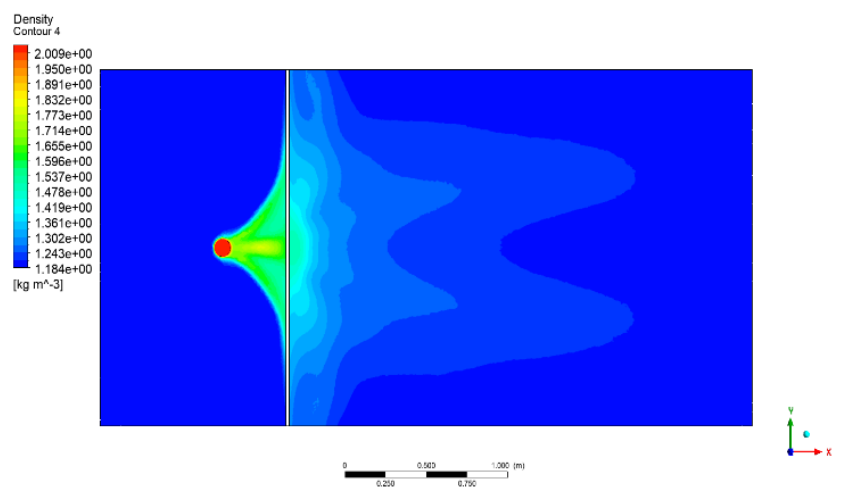

(4)

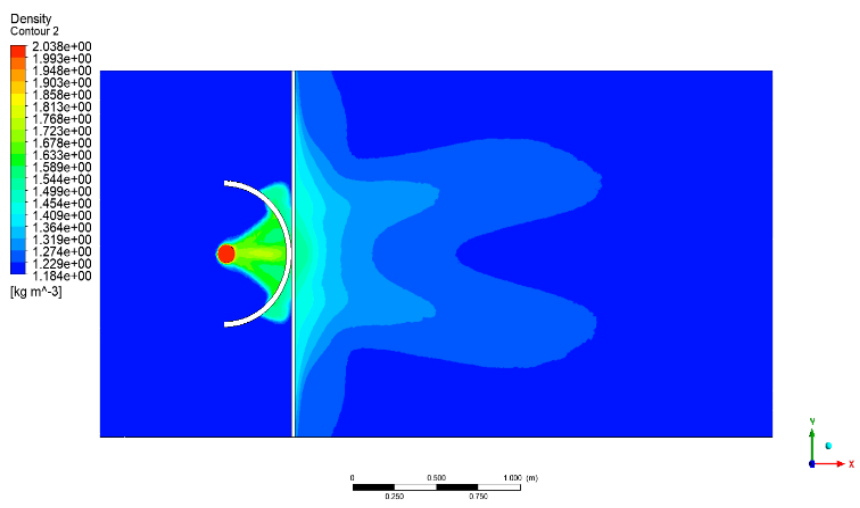

(5)

In order to visualize the shapes of heavy gas cloud, isosurfaces of the heavy gas concentrations are showed in Figure 5.

\section{Figure 5}

Visualization of 3D shape of the heavy gas clouds

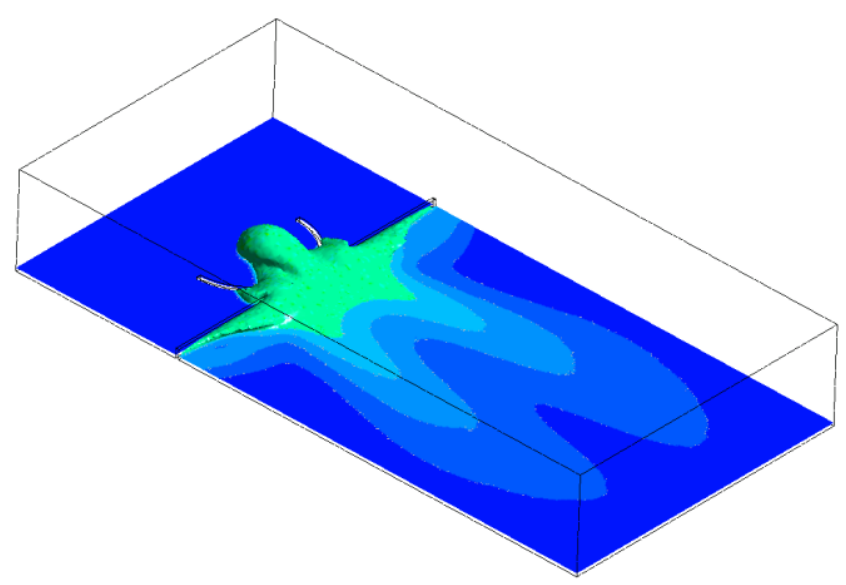

(1)

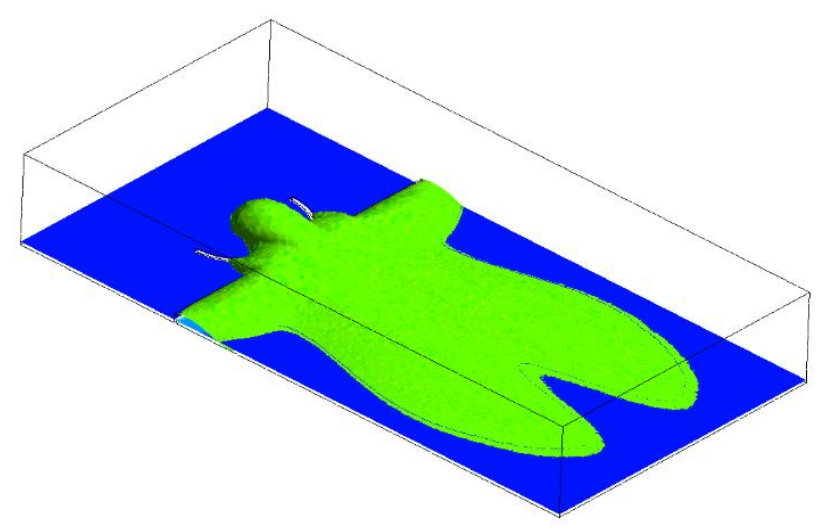

(2)

\section{Conclusions}

This work presents a numerical investigation into the heavy gas dispersion in the atmosphere through numerically solving the Reynolds averaged Navier Stokes equations. The numerical results show the double peaks pattern of the heavy gas concentrations. This kind of pattern is caused by the presence of buoyance. When obstacles exist, the heavy gas cloud shapes will significantly change. The higher the obstacles the more the heavy gas centralizes upstream. These results are in agreement with the experimental measurements and observations.

$\overline{\text { *Correspondence author: Abdullah Alakalabi, Email: aalakalabi@uclan.ac.uk }}$ 


\section{Acknowledgments}

The author has a scholarship from the Saudi Arabia government to do this project. However, the completion of this piece of work could not have been possible without the participation and assistance of the director study Dr. Weiming Liu, which are sincerely appreciated and gratefully acknowledged.

I would like to thank and appreciate the University of Central Lancashire-UCLan for providing the ANYASCFX software tools, which helped me to obtain such accurate results.

\section{References}

1. Launder, B E and Spalding, D B, 1974, The numerical computation of turbulent flows, Computer Methods in Applied Mechanics and Engineering, Vol. 3, 269 - 289. 2. Liu, W, Alakabi, A, Graham, T and Gu, X, Large eddy simulations of heavy gas dispersion within building group, ICCFD9 Proceedings, 2016, ICCDF9-2016-136

3. ANSYS, ANSYS CFX-Solver Theory Guide, Release 19.1, April 2018, ANSYS Inc

4. Liu, W. and Makhviladze, G, 2008, An implicit finite element solution of thermal flows at low Mach number, Journal of Computational Physics, Vol 227, 2743 - 2757. 5. Ayrault, M., Simoens, S. \& Mejean, P, 1994,

Dispersion of Dense Gas Releases Downwind of Solid Obstacles. Transactions on Ecology and the Environment. 4: 491-498.

6. Ayrault, M., Simoens, S. \& Mejean, P, 1997, Negative buoyancy effects on the dispersion of continuous gas plumes downwind solid obstacles. Journal of Hazardous Materials. 57: 79-103. 